\title{
Study on Fragment Velocity Measurement Technology of Prefabricated Fragment Warhead
}

\author{
Zhitong Yu ${ }^{1, a,}{ }^{*}$ and Dongmei Shi ${ }^{1}$ \\ ${ }^{1}$ Shijiazhuang Campus, Amy Engineering College, Shijiazhuang Hebei 050003, China \\ 1640567274@qq.com
}

Keywords: Prefabricated fragment warhead; velocity measurement technique; contact velocity measurement; non-contact velocity measurement.

\begin{abstract}
The prefabricated fragment warhead has been widely used in the military field at home and abroad in recent decades because of its characteristics such as regularity of fragment shape formation, good dispersion characteristics, and strong anti-destructive capability. Among them, the fragment velocity is an important indicator to measure its damage ability; fragment velocity measurement methods can be divided into contact velocity measurement method and non-contact velocity measurement method. This paper focuses on the analysis and comparison of the technical status, advantages and disadvantages of these two methods, and summarizes and forecasts the fragment velocity measurement methods of prefabricated fragment warheads.
\end{abstract}

\section{Introduction}

In the existing conventional weapons, most of the warheads use fragment destruction methods. For example, warheads such as air defense, anti-radiation, and surface killing generally use fragment warheads, relying mainly on fragment kinetic energy to destroy targets. The Fragmentation Warhead is one of the main types of warheads. It is mainly due to the explosive action of high-energy explosives. It forms a large number of high-speed fragments and uses the fragment's high-speed impact, ignition and detonation to damage the target. It can be used to kill and kill force (human, livestock), non-armoured or light armored vehicles, aircraft, radar and missiles, etc. ${ }^{[1]}$

According to the fragment generation method, the fragmentation warheads can be divided into three types: natural, pre-control, and prefabricated fragment warheads. The fragments of natural fragment warheads are formed by the expansion and fracture of the shell under the action of detonation products. The characteristics of this type of warhead are that the shell acts as both a container and a killing element, and the utilization of materials is high. Thicker, before the detonation product leaks, the driving acceleration time is long, and the formed fragment has a high initial velocity, but the size of the fragment is uneven, the shape is irregular, and the velocity decays quickly when flying in the air. The pre-control fragment warhead adopts technical measures such as shell notching, explosive notching or adding inner lining to weaken the local strength of the shell and control the ruptured part of the explosion to form a fragment. The characteristics of this type of warhead are that the fragments formed are uniform in size and 
have a basic shape. Fragments of prefabricated fragment warheads are pre-formed and embedded in the shell matrix material or bonded to the thin skin surrounding the explosive. Explosives explode to eject them. The fragments are tile-shaped, cubic, spherical, and short. Columns, etc., are characterized by the size and shape of the fragments. The explosive energy of the explosives is not used for fragmentation and fragmentation. The energy efficiency is high and the killing effect is good ${ }^{[2]}$.

Prefabricated structures have several major advantages:

1) The forming property is more superior, and the fragment can be processed into almost any desired shape to meet various flying characteristics requirements;

2) Fragments have better speed decay characteristics than other fragment warheads;

3) The prefabricated fragments can be processed into special types, for example, high-specific gravity materials can be used for fragmentation to improve the penetration ability; different fillers can also be installed inside the fragment to increase the fragmentation efficiency;

4) Special materials can be used as prefabricated fragments to increase their damage performance, such as amorphous alloy preforms.

The biggest difference between the prefabricated fragment and the traditional natural fragment is that the fragment formed after the explosion has regular shape, uniform quality and good consistency. Therefore, it is not necessary to consider the effects of different sizes and shapes when making speed measurements. In addition, because the mass and shape of a single fragment are determined, ie, the velocity attenuation law is consistent, only a single measurement point needs to be set to measure the velocity of the desired point during velocity measurement, and it is not necessary to verify the velocity attenuation rule of the dispersion.

The fragment measurement method can be divided into two categories according to whether the fragment and the sensor have direct physical contact: contact measurement and non-contact measurement ${ }^{[3]}$.

\section{Contact Velocity Measurement Method}

The contact velocity measurement method mainly includes aluminum foil target method, net target method, and comb target method ${ }^{[4]}$. Among them, the most typical and widely used is the aluminum foil target method. The basic measurement principle is that the aluminum foil target consists of two aluminum foil layers separated by a certain distance. When the fragment penetrates the front and back target plates, the aluminum foil layer is connected by the fragments to generate a pulse signal, and the system records this signal generation time. A set of aluminum foil targets are set in the flight direction of the fragment, and the speed variation of the fragment scattering process can be obtained by the distance between the target and the time difference. A single aluminum foil target can only be used to measure the fastest speed fragments and cannot be reused. However, due to the relatively low cost of aluminum foil target, the principle is simple, and the layout is convenient, the test accuracy can basically meet the requirements, so it is widely used in the measurement of fragmentation velocity of static explosion test.

Regardless of the aluminum foil target method, the net target method or the comb target method, the principle is relatively simple, and the technical approach is relatively mature. In recent years, there has been less research on technical principles in this area. Wang Gao ${ }^{[5]}$ proposed a resistance network target measurement method for the problem that the target can only measure the velocity of the first projectile or fragment. The essence of this method is to change the serial network cables of common network targets into parallel, and connect a resistor in series with each network cable. When the fragments pass through the target surface, any one of the network wires is interrupted will cause a change in the total resistance. According to the 
time interval and amplitude of the total resistance of the two targets, the speed of multiple fragments can be measured and the fragment size can be estimated. This method is a huge improvement to the net target method, but the accuracy is low, and the reliability of a single network cable is poor, and when the fragment passes through a failure region where a fragment has previously passed, secondary measurements cannot be performed.

The contact velocity measurement method is the earliest method to be applied to the fragment velocity measurement, and it still plays an irreplaceable role in the measurement of fragmentation velocity in the static burst test at home and abroad. The advantages are relatively low cost, simple installation and installation, and the test accuracy can basically meet the basic requirements of the fragmentation speed test of the target field. However, there are many limitations in the actual use process. If it is desired to achieve a good test effect, the single fragment generated by the explosion must be traversed along the linear trajectory to destroy all targets after it has been ejected from the blast center ${ }^{[6]}$. Obviously, it is difficult to meet this condition during the explosion process. The main disadvantages of this method are that it can only measure the fastest fragmentation rate, cannot be reused, has limited test accuracy, and is difficult to deploy a large number of test points at the same time.

The advantages and disadvantages of the contact velocity measurement method are as follows:

1) Prefabricated fragments are regular in shape and uniform in quality and do not have to be considered in this aspect when making measurements.

2) The contact measurement method generally can only measure the fastest fragment velocity of the flight speed; during the explosion process of the prefabricated fragment warhead, due to the general density of the prefabricated fragment, the initial velocity is likely to be lower than the natural fragment, but its quality rule and speed Slow decay, high speed. Therefore, it cannot be guaranteed that the measured speed is the speed of the prefabricated fragment.

3) Although the thickness, strength, and strength of the aluminum foil target plate, mesh target, and comb-shaped target plate are small, the direction, speed, and flight attitude of the high-speed flying fragment may be affected to a certain extent, and the measurement accuracy may be reduced.

4) The velocity distribution and fly angle of preformed fragments are important tactical indicators. However, due to the time-consuming and labor-intensive production and fabrication of aluminum foil targets, mesh targets, and comb targets, a large number of test points need to be installed to obtain accurate and complete data. time.

\section{Non-contact Velocity Measurement Methods}

Non-contact measurement methods include coil targets, sky screen targets, light curtain targets, and high-speed photographic methods ${ }^{[3]}$. Among them, the coil target and the sky screen target are mostly used for measuring the initial velocity of the projectile. The size of the fragment is small and the flight direction is not controllable. Therefore, it is rarely used in the fragmentation field; if it is widely used in the field of fragment velocity measurement, the two methods must be greatly improved. Accuracy and the ability to measure multiple targets simultaneously.

Nowadays, rapid progress and the use of fragmentation in the measurement of velocity are high-speed photographic methods and light curtain methods. The high-speed photography method is an advanced speed measurement method, which can not only aim at a single fragment emitted by a specific launch device, but also measure the speed condition of the fragment group during the explosion process ${ }^{[7]}$. The light curtain target test system usually includes four parts of 
a velocity screen target (photoelectric sensor), a signal conditioning circuit, a data acquisition card, and a processing computer ${ }^{[8]}$. The basic principle is that the laser beam emitted by the laser diode array is irradiated to the photodiode array surface to form a laser curtain. When the fragment passes through the light curtain, it forms a block to generate a pulse signal, and the data acquisition system records the time when the fragment passes. The average velocity of the fragment passing through the two light curtains can be calculated by the distance between the two light curtains and the time difference of the fragments passing through the light curtain. Light curtain targets are currently only used for fragment velocity measurements emitted by certain launch devices, such as the velocity measurement section of experimental devices such as light gas cannons and explosive chambers. Due to difficulties in protection, non-fragmentation interference, and limited laser screen formation, it is temporarily impossible to measure the velocity of fragments in static explosion tests.

The non-contact speed measurement method has high accuracy because of its fast response speed; and when non-contact measurement is performed, the fragment has no substantial damage to the target itself and can be reused; the high-speed photography method can also roughly measure the speed and distribution of the fragment group. . For prefabricated fragment warheads, light curtain targets are often used to test the velocity decay characteristics of a single prefabricated fragment, or to verify the correspondence between individual fragment velocity and penetration properties, and cannot be used in pre-fabricated fragment warhead static burst test fragment group velocity measuring. Also, fragmentation will have a certain scattering angle during the flight, so there will be some deviation in the test.

At present, in-depth non-contact speed measurement has been conducted in-depth research at home and abroad. Among them, Zhang Huwei and Li Jinming of North China University ${ }^{[9]}$ designed a non-contact two-dimensional light to meet the test difficulties of multiple targets, fast speed, small size, high light intensity, and strong electromagnetic interference in the test environment. Curtain fragment kinetic energy test system. The system solves the problem that the traditional one-dimensional light curtain test system can only measure the kinetic energy of rotationally symmetric fragments. The use of two-dimensional orthogonal light curtains to effectively measure the kinetic energy information of non-rotationally symmetric fragments has certain practical value and application prospects. Ni Jinping and Tian Hui et al. ${ }^{[10]}$ proposed a method for velocity measurement using multiple light curtain targets, data acquisition instruments, and position markers. The size and number of fragments can be roughly identified based on the collected fragment fragmentation target waveforms. Wei Hongqiang and Wang Jinsong et al ${ }^{[11]}$ proposed a non-contact velocity measurement method for warhead fragments of warheads based on laser target technology. This method can automatically identify the overshoot target position and the actual flight direction velocity of a single fragment in a fragment group. It can accurately measure the flight speed of the fragment group, which is conducive to accurate calculation of the fragment initial velocity and the fragment velocity attenuation coefficient.

\section{Conclusion and Outlook}

Because of its unique advantages such as high energy utilization rate and strong damage capability, the prefabricated fragment warhead has been developed and used for combat at home and abroad in recent decades. Therefore, the method of measuring the velocity of a prefabricated fragment during the development of an experiment also tends to be more mature and perfect. However, due to the short explosion process and strong destructive power of the prefabricated fragment warhead, the static explosion test in the field test field still uses the contact velocity measurement method. Non-contact velocity measurement methods are used in the study of 
velocity measurements and attenuation characteristics of individual fragments.

With the rapid development of sensor technology, array camera high-speed photography technology, image processing technology, machine vision technology and laser screen technology, non-contact velocity measurement methods will be increasingly used in the field test field prefabricated fragment group velocity and other parameters measurement In addition, it may gradually replace the contact speed measurement method.

\section{References}

[1] Han Fuliang. Numerical simulation analysis of explosive power field of prefabricated fragment warhead[D]. Jilin: Master thesis of Jilin University, 2008.4:1-5.

[2] Zhao Yudong, Jin Li, Yan Yulan et al. Analysis of Influencing Factors of Prefabricated Fragment Killing [J]. Xi'an: Chinese Journal of Archery and Missile Command, 2007, 27(2) :50-51.

[3] Ni Jinping, Yang Lei. Velocity Measurement Method for Identifying Fragment Group Flight Direction and Position [J]. Chinese Journal of Ordnance. 2007 (01).

[4] Xu Dixin. Application of target net test method in fragment initial velocity test [J]. Modern Measurement and Laboratory Management. 2012 (01).

[5] Wang Gao, Yin Guoxin, Li Yangjun, Zhou Chengxian, Feng Qiaoling. Velocity Measurement Method of Fragmentation Group of Resistance Network Target [J]. Journal of Detection and Control. 2011 (03).

[6] Yang Yantong, Long Hongming. Error Analysis of Missile Warhead Fragmentation Kill Test [J]. National Defense Technology Foundation. 2003 (03).

[7] Shen Mande. Research on Fragment Parameter Precision Measurement Technology Based on Computer Vision [D]. Graduate University of Chinese Academy of Sciences (Xi'an Institute of Optics and Fine Mechanics). 2008.

[8] Huang Zhengxiang, Gao Senlie, Li Liangwei, Chen Huiwu. Study on Velocity Measurement Method of Small Caliber Projectile Fragments[J]. Journal of Nanjing University of Science and Technology. 2000 (05).

[9] Zhang Huwei, Li Jinming, Gao Wengang, Guo Wei. Design of two-dimensional light curtain fragment kinetic energy test system [J]. China Test. 2016 (08).

[10] Ni Jinping, Tian Hui, Yang Lei. Research on Measuring Method of Fragmentation Speed of Light Curtain Target in Warhead [J]. Optics Technology. 2008 (01).

[11] Wei Hongqiang, Wang Jinsong, Feng Jinliang, Ma Hong, Li Shuhua, Song Xiaohui. Velocity Measurement Method for Fragment Group of Warhead Based on Laser Target [J]. Chinese Journal of Scientific Instrument, 2008. 